\title{
La Borysthène archaïque (site de l'île de Bérézan)
}

Première colonie grecque du nord de la mer Noire, d'après le matériel des fouilles récentes du Musée d'état de l'Ermitage menées dans la partie orientale de l'île

\section{Dmitrij Efimovič Čistov}

Traducteur : Mathilde Reichler Imperiali

\section{OpenEdition}

\section{Journals}

Édition électronique

URL : http://journals.openedition.org/edl/356

DOI : 10.4000/edl.356

ISSN : 2296-5084

Éditeur

Université de Lausanne

Édition imprimée

Date de publication : 15 mai 2012

Pagination : 223-260

ISBN : 978-2-940331-27-7

ISSN : 0014-2026

\section{Référence électronique}

Dmitrij Efımovič Čistov, « La Borysthène archaïque (site de l'île de Bérézan) », Études de lettres [En

ligne], 1-2 | 2012, mis en ligne le 15 mai 2015, consulté le 18 décembre 2020. URL : http:// journals.openedition.org/edl/356 ; DOI : https://doi.org/10.4000/edl.356 


\title{
LA BORYSTHÈNE ARCHAÏQUE (SITE DE L'ÎLE DE BÉRÉZAN)
}

\author{
PREMIÈRE COLONIE GRECQUE DU NORD DE LA MER NOIRE, \\ D'APRÈS LE MATÉRIEL DES FOUILLES RÉCENTES DU MUSÉE D'ÉTAT DE \\ L'ERMITAGE MENÉES DANS LA PARTIE ORIENTALE DE L'ÎLE
}

Il semble que l'établissement de Bérézan, qui portait dans l'Antiquité le nom de Borysthène, puisse être considéré comme l'un des monuments clefs de l'archéologie antique du nord de la mer Noire. Dans les couches anthropiques de l'établissement de la période archaïque étudiées jusqu'à aujourd'hui, on distingue deux phases principales: la première, qui s'étend de la fin du VII ${ }^{\mathrm{e}}$ siècle jusqu'au milieu du VI ${ }^{\mathrm{e}}$ s. av. J.-C., est constituée exclusivement de structures d'habitation de type "cabanes semi-enterrées» ainsi que de fosses domestiques. C'est dans les années 540 av. J.-C. environ qu’apparaît à Bérézan un site urbanistique: un réseau de rues et de quartiers, avec des maisons «en surface», faites de pierre et de brique crue. Manifestement, ces changements frappants furent occasionnés par l'arrivée d'une nouvelle vague de colons, après la soumission des villes ioniennes à l'Empire perse. Un établissement de type urbain se maintint à Bérézan approximativement jusqu'à la fin du premier quart du Ve s. av. J.-C., ensuite de quoi il fut abandonné par les habitants. De nouvelles fouilles, entreprises par l'expédition de l'Ermitage dans la partie orientale de l'île, montrent que cette phase «urbanistique» peut être à son tour divisée en deux étapes délimitées par les traces d'importants incendies et de destructions qui eurent lieu au cours du dernier quart du $\mathrm{VI}^{\mathrm{e}} \mathrm{s}$. av. J.-C. Le tournant des $\mathrm{VI}^{\mathrm{e}}-\mathrm{V}^{\mathrm{e}} \mathrm{s}$. av. J.-C. marque l'étape finale de l'existence de la ville archaïque; à cette époque apparaît un nouveau complexe d'édifices publics dans la partie orientale de l'établissement: il pourrait s'agir d'un téménos, partiellement mis au jour lors des fouilles de ces dernières années.

Bérézan, l'une des quelques îles que compte la mer Noire, est située dans l'embouchure de l'estuaire du Dniepr et du Boug méridional. Malgré une superficie très réduite (l'île s'étend aujourd'hui sur près de $850 \mathrm{~m} \mathrm{du}$ nord au sud et de $480 \mathrm{~m}$ d'est en ouest), Bérézan est un site exceptionnel 
au niveau historique et archéologique: elle concentre sur son territoire des couches d'occupations et des vestiges de constructions d'un très large spectre de périodes historiques, de l'époque archaïque (seconde moitié du VII s. av. J.-C.) aux années quarante du XXe siècle. Le paysage actuel de l'île porte dans une large mesure la trace de l'action de l'homme: il est le résultat de plus de deux mille cinq cents ans d'une intense activité humaine. Ainsi, bien que Bérézan soit aujourd'hui privée de source d'eau douce, inhabitée et dépourvue d'infrastructures touristiques, peu de sites ou de territoires lui sont comparables en termes de concentration de vestiges du patrimoine historique d'époques, de cultures et de peuples divers.

On considère traditionnellement Borysthène (l'établissement de Bérézan) comme un monument clef de l'archéologie antique du nord de la mer Noire, dans la mesure où elle apparaît comme le centre le plus ancien de la civilisation grecque de cette région. L'île a attiré l'attention des scientifiques spécialistes de l'Antiquité dès les années vingt du $\mathrm{XIX}^{\mathrm{e}}$ siècle et des fouilles systématiques y ont été engagées il y a plus de cent ans. Le territoire de Bérézan fait aujourd'hui partie du Parc national archéologique et historique "Olbia» de l'Académie nationale des sciences d'Ukraine. Des expéditions de l'Institut d'archéologie de l'Académie nationale d'Ukraine (Kiev) et du Musée de l'Ermitage (SaintPétersbourg) y réalisent des fouilles chaque année depuis le début des années 1960. Au cours des cinquante dernières années, les archéologues de l'Ermitage ont mené des recherches en plusieurs points de l'établissement, mais depuis 1998 leurs efforts se concentrent sur le secteur «O (fig. 1), situé dans la partie NE de Bérézan. C'est à un tour d'horizon des principaux résultats de ces récentes recherches qu'est consacré cet article.

Le secteur " $\mathrm{O}$ " possède une longue histoire dans la recherche archéologique. Dans cette partie de l'établissement, située sur une haute berge escarpée, non loin d'un promontoire orienté vers l'estuaire du liman de Bérézan, les fouilles furent engagées en 1960 par l'archéologue de Kiev Vladimir Vasil'evič Lapin ${ }^{1}$, qui les poursuivit jusqu'à ce qu'elles soient brutalement interrompues par son décès prématuré en 1980. A

I. L'abréviation « $\mathrm{O}$ » qui a cours actuellement vient du mot russe "osnovnoj" ("principal») : durant deux décennies, ce secteur était la principale zone de fouilles de Vladimir Vasil’evič Lapin. 
Fig. 1 - Plan topographique de l'île de Bérézan (réalisé par le géodésiste D. Belen'kij). La flèche indique l'emplacement du secteur de fouilles « $\mathrm{O}$ ». 
plusieurs reprises avant le début des travaux de l'expédition ukrainienne, cette zone avait été l'objet de recherches archéologiques; à proximité immédiate se trouvent les secteurs explorés par Ernst Romanovič fon Štern en 1904 et par Mikhail Fjodorovič Boltenko en 1946-1947. Les fouilles systématiques menées par Lapin durant deux décennies ont permis de mettre au jour un secteur d'habitations de la Borysthène archaïque des $\mathrm{VI}^{\mathrm{e}}$ et $\mathrm{V}^{\mathrm{e}}$ s. av. J.-C. de dimensions tout à fait considérables. Les recherches archéologiques dans cette partie de l'île furent reprises en 1990 à l'initiative de Jurij Germanovič Vinogradov, célèbre spécialiste de l'Antiquité, qui entreprit quelques fouilles mineures dans la zone de la "maison à abside» découverte par Lapin, un intéressant complexe qui semble avoir été un bâtiment public, dédié au culte ou à destination civile. C'est précisément cette zone qui fit par la suite l'objet de fouilles systématiques de l'expédition de l'Ermitage, dont les travaux se poursuivent encore à l'heure actuelle. La superficie totale étudiée du secteur " $\mathrm{O}$ " s'étend aujourd'hui sur près de $4600 \mathrm{~m}^{2}$ (le secteur exploré par Lapin dans les années 1960-1980 atteignait $3000 \mathrm{~m}^{2}$ ).

La période primitive (fin du VII $I^{e}$ première moitié du VI ${ }^{e}$ s. av. J.-C.)

L'établissement de Bérézan est l'une des premières colonies de la côte septentrionale de la mer Noire. La Chronique d'Eusèbe de Césarée mentionne la fondation de Borysthène en 647-646 av. J.-C. (Chron. Com. 95b Helm 1984), mais la datation exacte du premier établissement permanent sur l'île de Bérézan reste encore sujette à débat aujourd'hui. Cela est dû à l'absence, à l'heure actuelle, de complexes archéologiques bien documentés (habitats: cabanes semi-enterrées ${ }^{2}$, fosses domestiques, etc.) et datés d'une époque antérieure à la fin du VIIe siècle ou au tournant des VII ${ }^{\mathrm{e}}-\mathrm{VI}^{\mathrm{e}}$ s. av. J.-C.; ainsi, sur ce point, "la mention d'Eusèbe entre en contradiction irréductible avec les données archéologiques" ${ }^{3}$. Bien que la présence de colons grecs à Bérézan dans le troisième quart du VII siècle soit indirectement confirmée par la découverte de fragments

2. «Dugout» et «semi-dugout» dans la littérature scientifique anglo-saxonne. Voir aussi, dans le présent volume, l'article de Jurij Vinogradov (Ndlt).

3. S. L. Solov'jov, "Načalo antičnoj èpokhi v Severnom Pričernomor'e», p. 9. 
de céramique de cette époque ${ }^{4}$, la quantité totale de telles trouvailles reste pour l'instant très faible. Cette contradiction pourrait s'expliquer par l'érosion côtière, qui a détruit les couches les plus anciennes de la zone littorale de l'île, de même que par le fait que l'établissement n'a été exploré encore que de manière incomplète 5 . Il semblerait logique de localiser la zone la plus ancienne de l'établissement dans la partie ouest et nord-ouest de l'île, cette zone étant la plus basse (l'horizon des cabanes semi-enterrées de la première moitié du $\mathrm{VI}^{\mathrm{e}}$ s. av. J.-C., dans le secteur " $\mathrm{O}$ », se trouve à près de huit mètres au-dessus du niveau de la mer) et pourvue en sources d'eau douce, ce dont témoignent les nombreux puits antiques que les fouilles ont permis de mettre au jour. Des doutes justifiés ont été émis quant à la légitimité de l'explication de la discordance entre la source écrite et les données archéologiques concernant la période la plus ancienne de l'existence de l'établissement par le fait que ces dernières sont trop lacunaires ${ }^{6}$. En effet, la côte orientale de l'île a été la moins soumise aux processus d'érosion, tandis que les fouilles sous-marines n'ont pas permis de mettre au jour d'objets en céramique si anciens ${ }^{7}$. D'un autre côté, la question de la dynamique de croissance de l'établissement dans la période de la fin du VIIe siècle à la moitié du $\mathrm{VI}^{\mathrm{e}}$ s. av. J.-C. n'ayant pour l'instant pratiquement pas été étudiée, on peut tout de même supposer que la zone la plus ancienne n'a pas encore été découverte par les archéologues: la surface occupée par les premiers colons pour leurs habitations et leurs besoins domestiques devait être sensiblement plus réduite que celle du territoire de l'établissement durant la première moitié du $\mathrm{VI}^{\mathrm{e}} \mathrm{s}$. av. J.-C.

Létablissement archaïque de la période primitive avait une apparence bien caractéristique: jusqu'à la moitié ou jusqu'au début du troisième quart du $\mathrm{VI}^{\mathrm{e}}$ s. av. J.-C., le principal type d'habitation et de constructions domestiques des habitants de Borysthène était des bâtisses enfouies

4. L. V. Kopejkina, «Rodossko-ionijskaja keramika VII v. do n.è. s ostrova Berezan' i ejo značenie dlja izučenija rannego ètapa suščestvovanija poselenija", p. 71; J. I. Il'ina, "O novykh nakhodkakh vostočno-grečeskoj keramiki na ostrove Berezan'», p. 201-203.

5. J. G. Vinogradov, J. V. Domanskij, K. K. Marčenko, «Sources écrites et archéologiques du Pont Nord-Ouest».

6. S. L. Solov'jov, "Načalo antičnoj èpokhi v Severnom Pričernomor'e», p. 9.

7. V. V. Nazarov, Gidroarkheologičeskaja karta černomorskoj akvatorii Ukrainy, p. $43-53$. 
dans le sol, dites cabanes semi-enterrées ou enterrées. Elles étaient de formes diverses: les fosses d'implantation sont en général rectangulaires, ovales ou circulaires. Ces constructions possédaient un intérieur de la plus grande simplicité et des structures de toit conique, pour les circulaires et les ovales, ou à un ou deux pans, pour les rectangulaires ${ }^{8}$. Les dimensions de ces habitations étaient très réduites, la surface de beaucoup d'entre elles ne dépassant pas 8 à $9 \mathrm{~m}^{2}$. Bien qu'aucun indice clair d'agencement de l'établissement de la première période n'ait pu être relevé, on observe que les cabanes semi-enterrées étaient regroupées «en buissons" ou en chaînes constituées de quelques unités (pl. XVII.1 et fig. 2).

Les cabanes semi-enterrées de la première moitié du VI $\mathrm{VI}^{\mathrm{e}}$ s. av. J.-C. découvertes durant les deux dernières décennies dans le secteur " $\mathrm{O}$ " partagent beaucoup de points communs sur le plan de la construction. Le type dominant était la cabane semi-enterrée de plan arrondi (21 des 32 complexes étudiés ont un plan arrondi, neuf d'entre eux sont de forme quasi rectangulaire, l'état de conservation des deux derniers étant trop mauvais pour que l'on puisse déterminer leur plan). Les cabanes semi-enterrées circulaires (fig. 3.1-6), dans leur majorité, sont des excavations peu profondes $(20-60 \mathrm{~cm})$ pratiquées dans l'humus ancien et les colluvions naturelles sous-jacentes, d'un diamètre de 2,4 $\mathrm{m}$ à $3,3 \mathrm{~m}$ (c'est-à-dire d'une surface de 4,5 à $8,5 \mathrm{~m}^{2}$ ), aux parois verticales ou s'arrondissant progressivement à leur base. Deux cabanes arrondies étaient de dimensions légèrement supérieures, avec des diamètres de 3,4 $\mathrm{m}$ et 4,4 $\mathrm{m}$. Toutes les constructions mentionnées ci-dessus sont dépourvues de traces de foyers ou de braseros. Dans toutes les cabanes circulaires, à l'exception d'une seule, un trou de poteau central a été mis au jour, ce qui pousse à imaginer l'existence d'une simple couverture conique (cette cavité centrale est d'ailleurs souvent unique). Dans certains cas (les constructions semi-enterrées $n^{\text {os }} 28,29$ et 33), on observe plusieurs trous de poteaux rapprochés ou qui se chevauchent les uns les autres, ce qui peut témoigner d'une deuxième ou troisième rénovation de la couverture ayant entraîné un repositionnement du poteau central. Parfois, plusieurs trous de poteaux circulaires ou des cavités de forme irrégulière sont observables près de la paroi ou au bord de la fosse d'implantation

8. S. N. Mazarati, V. M. Otreško, «Poluzemljanki i zemljanki», p. 8; D. E. Čistov, «K voprosu o poluzemljankakh arkhaičeskoj Berezani», p. 379 sq. 
Fig. 2 - Plan du groupe de cabanes semi-enterrées circulaires mis au jour en 2005 dans la partie nord du secteur « $\mathrm{O}$ ».

de la cabane, qui pouvaient servir à maintenir l'extrémité de perches soutenant un toit pyramidal. Le nombre total des trous de poteaux atteint dans certains cas le chiffre de neuf (cabane $n^{\circ} 37$ ). L'une des constructions circulaires ( $n^{\circ} 27$, fig. 2) était manifestement de facture plus complexe, avec des murs peu élevés en torchis montés sur treillis, dressés sur des poteaux de soutènement placés au bord de la fosse de construction. Les traces de ce mur en torchis et clayonnage se présentent sous la forme d'une rigole circulaire peu profonde creusée le long de la paroi sur presque tout le pourtour de la fosse d'implantation. Le long de cette rigole, on observe les traces de poteaux de soutènement (sept cavités plus 
Fig. 3 - Plans et coupes de quelques cabanes semi-enterrées mises au jour dans le secteur « $\mathrm{O}$ ». 1-6: cabanes semi-enterrées arrondies de la première moitié du $\mathrm{VI}^{\mathrm{e}}$ s. av. J.-C.; 7 : cabane ovale et cabane arrondie du milieu du VIe s. av. J.-C. 
ou moins bien visibles). Les traces d'un trou de poteau central, entouré de rigoles mal conservées, témoignent de la structure conique de la toiture. En général, dans les constructions mises au jour, la configuration de l'entrée n'est pas non plus observable. On a le sentiment que la plupart des structures étudiées n'ont servi que très peu de temps. On observe parfois dans les constructions semi-enterrées ce qu'on appelle des «tablettes" et des "couches", qui sont des marches modelées dans le terrain argileux, de faible hauteur (de $15 \mathrm{~cm}$ à $35 \mathrm{~cm}$ depuis le sol) et qui longent la paroi (fig. 3.1, 2, 4, 6). Dans bien des cas, du fait de leur petite largeur (de $30 \mathrm{~cm}$ à $8 \mathrm{~cm}$ ), de telles constructions ne pouvaient faire office de couche. La taille relativement standardisée des fosses d'implantation pratiquées pour l'aménagement de ces habitats primitifs (ou constructions domestiques) attire l'attention. Il ne faut certes pas prendre un tel «standard de logement» comme preuve d'une réglementation des constructions, mais il est plus que probable que ce standard existait, dans les consciences des membres de la communauté des colons, en tant que norme culturelle établie qu'il convenait d'observer dans la vie de tous les jours. Selon toute vraisemblance, dans notre cas, la norme pour le diamètre de la cabane semi-enterrée était de 10 pieds (ce qui correspond à 3,2 m ou 3,5 m, selon que l'on prend comme unité le pied dorien ou le pied de Samos ${ }^{9}$ ). L'absence de foyers dans les constructions mises au jour ne permet pas de les définir sans équivoque comme étant des lieux exclusivement d'habitation, mais ne récuse pas du tout cette interprétation non plus. Le peu de surface exploitable de telles structures rendait difficile l'installation de foyers permanents, mais permettait tout à fait un chauffage par braseros, dont les traces ne se fixent pas au cours des fouilles.

Les constructions semi-enterrées de forme quasi rectangulaire se rencontrent surtout aux alentours de deux ateliers de fonderie du bronze (fig. 4), qui apparaissent eux-mêmes comme deux cabanes semi-enterrées rectangulaires de dimensions assez importantes (par exemple, l'une d'elles, la $\mathrm{n}^{\circ} 6$, s'étend sur une surface de $19 \mathrm{~m}^{2}$ pour une profondeur

9. A propos de la possibilité de l'usage du pied de Samos (pied égyptien) pour le tracé des structures semi-enterrées d'Olbia et de ses environs à l'époque archaïque tardive, voir A. V. Bujskikh, «Dejaki osoblyvosti planuval'noi struktury pizn'oarkhaičnykh poselen' Nyžn'ogo Pobužžja», p. 24 et "O standartakh linejnykh mer v stroitel'no kamenotesnom dele Khersonesa IV-II vv. do n.è.», p. 96. 
Fig. 4 - Schéma de situation dans le secteur « $\mathrm{O}$ » des complexes métallurgiques de la première moitié et du milieu du $\mathrm{VI}^{\mathrm{e}} \mathrm{s}$. av. J.-C.

de $1,10 \mathrm{~m}$ à $1,20 \mathrm{~m})$. Soulignons qu'on ne peut pas considérer cette configuration comme exceptionnelle pour l'établissement de Bérézan; ainsi, Sergej L'vovič Solov'jov a fait remarquer qu'on rencontre dans la partie méridionale du secteur nord-ouest des cabanes semi-enterrées de formes diverses, alors que $80 \mathrm{~m}$ plus au nord prédominent nettement des structures circulaires, regroupées en hameaux, vraisemblablement des domaines isolés dont les habitats semi-enterrés ont été régulièrement reconstruits au cours d'une certaine période ${ }^{10}$. Les cabanes semienterrées de forme quasi rectangulaire constituent également une partie importante des complexes découverts plus à l'est, sur le territoire du secteur «O» exploré par Lapin dans les années 1960.

Les thèses divergent quant au statut de Borysthène à l'époque primitive et aux raisons de sa fondation. Selon l'une d'elles, cette apoikia

Io. S. L. Solovyov, Ancient Berezan, p. 41. 
doit être considérée comme un emporion (un comptoir de commerce) né du résultat de l'expansion économique de Milet au nord de la mer Noire et passé sous le contrôle d'Olbia après la fondation de cette dernière ${ }^{11}$. Un autre point de vue sur cette question consiste à considérer Borysthène comme une polis, une communauté de citoyens, ou même comme une structure politique plus ou moins autonome dès le moment de sa fondation ${ }^{12}$. Les tenants d'une troisième hypothèse avancent qu'au cours d'une première étape (jusqu'au troisième quart du VIe s. av. J.-C.), une longue période de «reconnaissance» de la région et de ses ressources par un tout petit groupe de marins et de marchands a précédé la colonisation massive de Bérézan; sur ce même territoire fut fondée, dans la période suivante, une polis, qui progressivement, vers le milieu du $\mathrm{V}^{\mathrm{e}} \mathrm{s}$. av. J.-C., intégra la communauté de citoyens de la polis voisine d'Olbia ${ }^{13}$. La question de la composition ethnique de la population de Bérézan ne fait pas non plus l'unanimité aujourd'hui parmi les spécialistes de l'établissement. Alors que le noyau des colonisateurs était formé de Grecs d'Ionie, le fait que des habitats enfouis aient été en usage, de même que la découverte de céramique modelée d'un type particulier dans les strates et dans les habitations de Bérézan, sont des arguments en faveur de la présence de groupes de populations barbares locales, majoritairement des ressortissants de la Scythie de la forêt-steppe ${ }^{14}$. D'après Solov'jov, ce sont précisément ces populations qui ont pu constituer la base des habitants permanents aux premiers temps du peuplement, alors que les colons, marchands et pionniers grecs y étaient minoritaires. Il convient de souligner que le principal argument de poids, bien que très indirect, en faveur de l'appartenance «barbare» des structures semi-enterrées des premiers temps pourrait être donné par une différence significative de proportion entre la céramique modelée et la céramique tournée (ustensiles de table ou de cuisine) trouvée d'une part dans les habitats de l'étape primitive de l'établissement, celle des structures semi-enterrées, et, d'autre part, dans les habitats de la période suivante, celle de l'urbanisation; toutefois,

II. Parmi les travaux récents, voir A. V. Bujskikh, «Nekotorye polemičeskie zametki po povodu stanovlenija i razvitija Borisfena i Ol'vii v VI v. do n.è.», p. 146-149 et 164.

I2. J. G. Vinogradov, Političeskaja istorija Ol'vijskogo polisa, p. 62, 64 sq. et 67 sq.

I3. S. L. Solov’jov, "Načalo antičnoj èpokhi v Severnom Pričernomor'e», p. 10.

I4. S. L. Solov’jov, «O formirovanii gorodskoj i polisnoj struktury Berezanskogo poselenija», p. 41 . 
à en juger par le mobilier des fouilles récentes, ce chiffre reste soumis à l'incertitude statistique ${ }^{15}$.

Par ailleurs, ces dernières années, de nouvelles données ont confirmé que l'établissement de la fin du VII ${ }^{\mathrm{e}}$ siècle et de la première moitié $\mathrm{du} \mathrm{VI}^{\mathrm{e}}$ s. av. J.-C. était spécifiquement orienté vers le commerce et les matières premières (pl. XVIII). A l'heure actuelle, il a été découvert sur le territoire de l'établissement de Bérézan pas moins de douze complexes pouvant être liés à la métallurgie ou au travail des métaux, aussi bien du cuivre que du fer. La principale concentration de tels complexes s'observe aujourd'hui dans la partie orientale de l'établissement, dans la zone des fouilles « $O$ » (sept complexes, fig. 4). Les premiers du genre à avoir été découverts, par Lapin, se trouvaient précisément dans le secteur étudié. Le chercheur a décrit trois "bassins" arrondis et un rectangulaire, d'un diamètre de 3,20 m à $4 \mathrm{~m}$, creusés dans le niveau naturel à une profondeur allant jusqu'à $1,30 \mathrm{~m}$, et dans lesquels étaient fichés une énorme quantité de scories de fer, de charbon, etc. ${ }^{16}$. On peut supposer que des structures telles que ces bassins, à la surface recouverte d'oxydes de fer, étaient des complexes servant à la concentration du minerai. On y procédait également au grillage ou au lavage à l'eau du minerai. Cette méthode simple pour le débarrasser des impuretés minérales plus légères qu'il contient était largement en usage dans l'Antiquité ${ }^{17}$.

Les deux cabanes rectangulaires datant de la première moitié du $\mathrm{VI}^{\mathrm{e}}$ s. av. J.-C. mentionnées plus haut (n ${ }^{\text {os }} 6$ et 13 ), qui portent des traces de production de cuivre pur destiné au commerce, furent découvertes $20 \mathrm{~m}$ à $30 \mathrm{~m}$ plus à l'ouest, lors de fouilles dirigées par Jaroslav Vital'evič Domanskij et Konstantin Konstantinovič Marčenko. Dans leurs fosses d'implantation ont été mis au jour divers éléments de construction que les chercheurs mettent en rapport avec leur fonction productive: des excavations en forme de cuvette, des "bassins" portant dans leur fond des traces de l'action de températures élevées, de l'argile calcinée sur le sol, des vestiges d'un four à voûte en terre battue d'un diamètre de près de $80 \mathrm{~cm}$, etc. Dans le comblement de ces ouvrages, on a trouvé une très grosse quantité de scories, de morceaux de charbon de bois, d'éclats

I5. D. E. Čistov, «K voprosu o poluzemljankakh arkhaičeskoj Berezani».

I6. V. V. Lapin, "Raskopki poselenija na ostrove Berezan’ v 1960 g.», p. 46 sq.;

S. N. Mazarati, V. M. Otreško, «Poluzemljanki i zemljanki», p. 118.

17. J. F. Healy, Mining and Metallurgy in the Greek and Roman World, p. 144-148. 
Fig. 5 - Fragments d'objets en fer trouvés dans le comblement de la fosse d'implantation $\mathrm{n}^{\circ} 35$ du milieu du VI ${ }^{\mathrm{e}}$ s. av. J.-C. 1 : bris d’une épée (БЭ 2006 32/475); 2 : bris de la lame d'une serpe (БЭ 2006 32/474); 3 : fragments de la lame d'un couteau (БЭ 2006 32/477), 4-5: clous de navire (БЭ 2006 32/478, 479).

et même de lingots de cuivre (bronze arsénié) d'un poids allant jusqu'à $125 \mathrm{~g}^{18}$. Le comblement d'une excavation ( $\left.\mathrm{n}^{\mathrm{o}} 35\right)$ de dimensions importantes $(6,2 \mathrm{~m} \mathrm{x} \mathrm{6,4} \mathrm{m)} \mathrm{du} \mathrm{milieu} \mathrm{du} \mathrm{VI} \mathrm{e} \mathrm{s.} \mathrm{av.} \mathrm{J.-C.,} \mathrm{mise} \mathrm{au} \mathrm{jour} \mathrm{dans}$ la partie occidentale du secteur " $\mathrm{O}$ », contenait des couches de minerai de fer concentré ainsi que des fragments d'objets de fer de destinations diverses (fig. 5). De telles trouvailles témoignent du fait qu'il existait quelque part dans les environs, au deuxième quart et au milieu du $\mathrm{VI}^{\mathrm{e}} \mathrm{s}$. av. J.-C., en plus des ateliers de fonderie du bronze, des ateliers de production sidérurgique, y compris une forge, dans laquelle on produisait et réparait divers objets de fer ${ }^{19}$.

I8. J. V. Domanskij, K. K. Marčenko, «Bazovaja funkcija rannego Borisfena», p. 26.

I9. D. E. Čistov, «Novaja nakhodka fragmentov meča iz kompleksa serediny VI v. do n.è. na učastke "O" Berezanskogo poselenija". 
La répartition des traces de production métallurgique dans presque toutes les parties suffisamment explorées de l'île ${ }^{20}$ rend compte du fait que pratiquement tout le territoire de l'établissement pouvait être exploité par les artisans pour l'installation de leurs ateliers dès le tournant des $\mathrm{VII}^{\mathrm{e}}$ et $\mathrm{VI}^{\mathrm{e}}$ s. av. J.-C. et jusqu'au troisième quart du $\mathrm{VI}^{\mathrm{e}}$ s. av. J.-C. De plus, l'immense majorité de ces structures date précisément de la période primitive de l'établissement de Bérézan, celle des constructions semi-enterrées, ce qui confirme en grande partie l'hypothèse de Domanskij et Marčenko, selon laquelle la «fonction de base» de l'établissement était avant tout celle d'une colonie exploitant des matières premières ${ }^{21}$.

\section{Le site urbanisé de Bérézan (seconde moitié du VI ${ }^{e}$ s. - premier quart du $V^{e}$ s. av. J.-C.)}

Conformément à la périodisation la plus détaillée du site de Bérézan, établie par Solov'jov, l'histoire de l'établissement durant la période archaïque se divise en deux périodes principales, dont la charnière se situe au troisième quart du VI ${ }^{\mathrm{e}}$ s. av. J.-C. C'est à ce moment que surviennent des transformations très significatives dans l'aspect de l'établissement, en particulier le passage à la construction de maisons «en surface" faites en pierre et en brique crue, et l'émergence sur le territoire de Bérézan d'une structure urbanistique planifiée. A l'intérieur de cette seconde période, qui s'étend jusqu'à environ la fin du premier tiers du Ve s. av. J.-C., Solov'jov distingue deux étapes, dont il situe le tournant à la fin du $\mathrm{VI}^{\mathrm{e}}$ s. av. J.-C. ${ }^{22}$. A cette époque, l'agencement intérieur des quartiers et d'une grande partie des maisons d'habitation dont ils sont constitués est soumis à des transformations importantes, n'entraînant toutefois pas de modification significative dans l'agencement d'ensemble de la ville ${ }^{23}$. Le caractère des événements à l'origine

20. V. V. Krutilov, «Železodelatel'naja masterskaja s syrcovymi stenami na territorii temenosa Berezanskogo poselenija»; V. V. Nazarov, A. G. Kuz’miščev, «K probleme interpretacii odnoj pozdnearkhaičeskoj poluzemljanki na o. Berezan’»; J. V. Domanskij, «Raboty Berezanskoj èkspedicii».

2I. J. V. Domanskij, K. K. Marčenko, «Bazovaja funkcija rannego Borisfena».

22. S. L. Solov' jov, «Novye aspekty istorii i arkheologii antičnoj Berezani », p. 90.

23. S. L. Solovyov, Ancient Berezan, p. 8 et 79. 


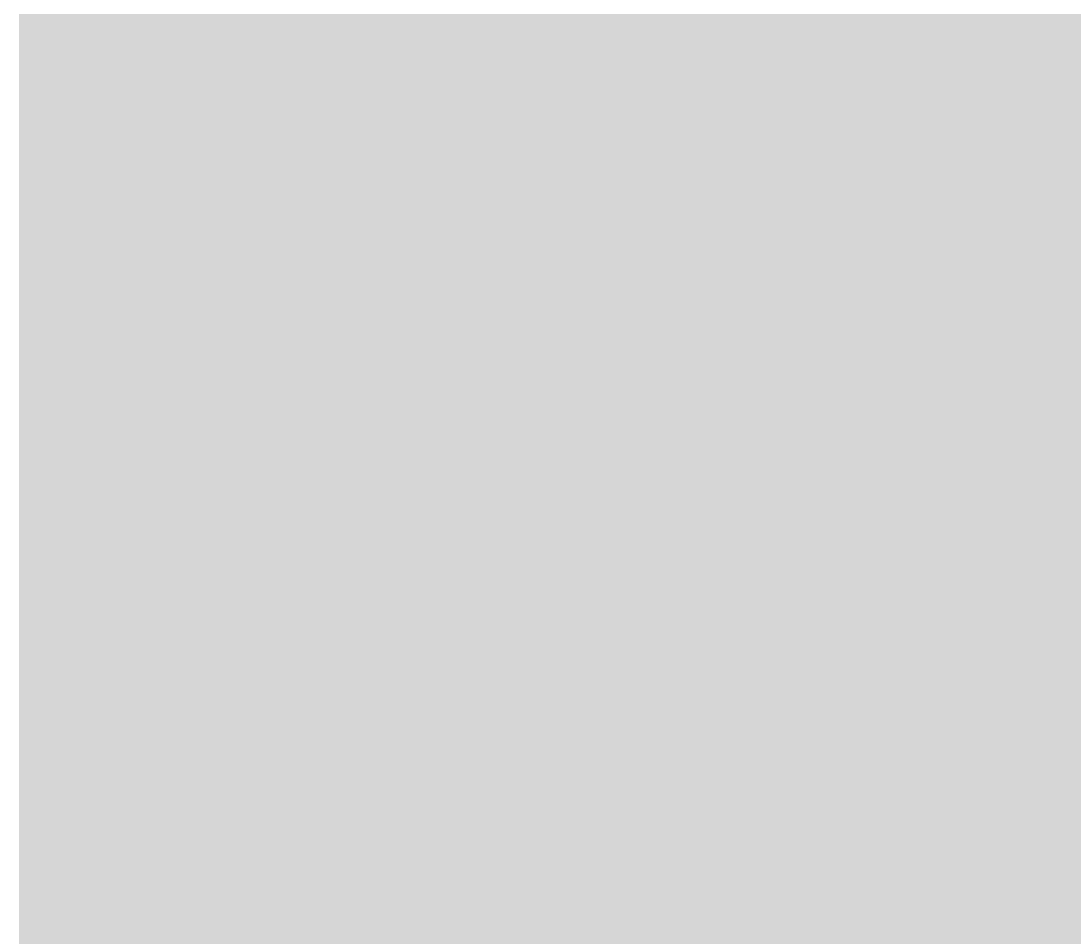

Fig. 6 - Secteur «O». Plan des vestiges de constructions de la seconde moitié du VIe s. av. J.-C.

de ces multiples reconstructions reste non éclairci. Les fouilles dans la partie nord-ouest de l'île n'ont permis de découvrir aucun témoignage de destruction ni d'acte guerrier. Ces constatations sont dans l'ensemble confirmées par les travaux de ces dernières années menés par l'expédition de l'Ermitage à Bérézan dans le secteur « $\mathrm{O}$ », dans la partie orientale de l'île, qui brossent un tableau analogue de l'évolution de Borysthène dans la seconde moitié du $\mathrm{VI}^{\mathrm{e}}$ s. av. J.-C. L'un des résultats de ces travaux a été la découverte d'une couche épaisse d'incendies et de destructions datant du dernier quart du $\mathrm{VI}^{\mathrm{e}}$ s. av. J.-C., révélée sur le territoire de deux quartiers urbains séparés par une rue (fig. 6). A l'est de cette rue, les vestiges d'une maison d'habitation détruite dans un violent incendie ont été partiellement explorés. A l'heure actuelle, deux pièces de cette maison ont été complètement mises au jour, ainsi qu'une portion d'une 
Fig. 7 - Plan des vestiges d'un bâtiment détruit lors d'un incendie au cours du dernier quart du $\mathrm{VI}^{\mathrm{e}}$ s. av. J.-C. 
cour clôturée par un mur de brique crue ${ }^{24}$. Du côté ouest, la cour intérieure était fermée par une annexe (partiellement explorée) aux murs de brique crue dépourvus de socle de pierre (fig. 7 et pl. XIX). Dans l'une des pièces du bâtiment $\left(\mathrm{n}^{\mathrm{0}} 5\right)$ a été découvert un foyer de construction intéressante: il s'agit d'une fosse rectangulaire aux parois enduites d'argile (pl. XX.1). Au fond de la fosse étaient placés deux fours portatifs de construction originale, faits d'argile crue sur une carcasse de baguettes cintrées (pl. XX.4). Ils avaient la forme de cylindres sans fond, avec deux poignées grossièrement modelées sur les côtés. L'un des fours reposait sur des pieds de petite taille, assurant un bon tirage pour une combustion normale du combustible à l'intérieur du cylindre (pl. XX.2); l'autre n'avait pas de pieds; ceux-ci étaient remplacés par trois pierres plates disposées en dessous. Une couronne supérieure aplatie en un épais bourrelet (pl. XX.3) permettait de poser sur ce genre de fours des pots de cuisine à fond arrondi typiques du $\mathrm{VI}^{\mathrm{e}}$ s. av. J.-C. La découverte de ce type d'objets, plutôt bien conservés, est unique pour le site de Bérézan; leur construction les rapproche toutefois de fours portatifs en céramique tournée connus d'après les fouilles de Milet ${ }^{25}$ et de l'agora d'Athènes ${ }^{26}$.

L'analyse du complexe céramique issu des couches de destruction (pl. XXI) a permis de préciser la datation du passage au mode de construction "en surface", que l'on peut situer, selon toute vraisemblance, au plus tard dans les années 540-530 av. J.-C.; la destruction par incendie du bâtiment, elle, est survenue au plus tard dans le dernier quart du VI ${ }^{\mathrm{e}}$ s. av. J.-C. Il est remarquable que les bâtisses plus tardives élevées à ce même emplacement après l'incendie s'inscrivent pratiquement dans le même plan que les précédentes; de toute évidence, elles se sont insérées dans le même système d'aménagement du quartier ${ }^{27}$. A certains endroits, elles on été érigées non pas sur les socles de pierre des murs précédents, mais par-dessus les amoncellements de brique crue des murs, qui ont selon toute apparence servi à leur manière de sousstructure. Il est clair que la reconstruction a débuté immédiatement après

24. D. E. Čistov, «Novyj kompleks s požariščem vtoroj poloviny VI v. do n.è. iz raskopok na učastke "O" antičnogo poselenija na o. Berezan" », p. 136, ill. 2.

25. A. Aydemir, "Funde aus Miletus XX», p. 94 sq., 99, Abb. 17, 18, cat. 8.

26. B. A. Sparkes, L. Talcott, Black and plain pottery of the 6 th, 5 th, and 4 th centuries B.C., pl. 97, n ${ }^{\text {os }} 2017$ et 2018.

27. D. E. Čistov, «Novyj kompleks s požariščem vtoroj poloviny VI v. do n.è. iz raskopok na učastke "O” antičnogo poselenija na o. Berezan'”, p. 133. 
l'incendie et qu'elle n'a pas été précédée de sérieux travaux préparatoires de nivellement de la surface sous la nouvelle bâtisse.

On peut supposer que les traces d'incendies et de destructions étudiées dans le secteur " $\mathrm{O}$ " ne sont pas des phénomènes locaux, et que le passage d'une étape à l'autre durant la deuxième période de la Borysthène archaïque a été marquée non seulement par l'intensification dans l'activité de construction de ses habitants, comme on le pensait déjà auparavant, mais également par certains événements ou catastrophes. Les causes de ce phénomène restent pour l'instant incertaines. Solov' jov supposait dans un premier temps un lien entre ces événements et l'arrivée d'une nouvelle vague de colons venus de la métropole ${ }^{28}$, mais il a également fait remarquer dans un récent article qu'il serait tentant d'y voir une répercussion du conflit entre deux groupes de colonisateurs, conflit tranché par l'oracle d'Apollon à Didyme ${ }^{29}$. Ce conflit, qui aurait pris la forme d'une opposition entre les adeptes du culte d'Apollon-Médecin et de celui d'Apollon-Delphinien ${ }^{30}$, aurait d'ailleurs conduit, d'après l'auteur, à la fondation de la polis d'Olbia sur des territoires antérieurement sous le contrôle de Borysthène ${ }^{31}$. Les nouvelles données, bien qu'elles ne soient à même ni de confirmer ni de réfuter les hypothèses rapportées plus haut, témoignent plutôt en leur défaveur, nous semble-t-il. Dans le cas contraire, il faudrait supposer que la confrontation entre des groupes relativement peu nombreux de colons et d'epoikoi aurait dégénéré en conflit armé, dont les colons vivant sur l'île même ne seraient clairement pas sortis vainqueurs, puisque les combats auraient gagné leur territoire. Quoi qu'il en soit, le tableau des destructions et des incendies relevés pour le dernier quart du $\mathrm{VI}^{\mathrm{e}} \mathrm{s}$. av. J.-C. dans différentes parties de l'agglomération archaïque permet de supposer que l'établissement de Bérézan a subi à cette époque des événements catastrophiques, peut-être une attaque ennemie, mais qu'il s'en est rapidement relevé.

Les travaux de l'expédition du Musée de l'Ermitage sur l'île de Bérézan ont produit ces dernières années des données totalement nouvelles, concernant directement la question du passage d'une étape à

28. S. L. Solov'jov, "O formirovanii gorodskoj i polisnoj struktury Berezanskogo poselenija", p. 41.

29. S. L. Solov’jov, «Iz istorii polisov Nižnego Pobuž ja», p. 102.

30. Sur la découverte du texte de l'oracle et sa lecture, voir A. S. Rusjaeva, «Milet Didimy - Borisfen - Ol'vija».

3I. S. L. Solov’jov, «Iz istorii polisov Nižnego Pobuž ja», p. 101. 
l'autre durant la deuxième période de l'existence de Borysthène. Au cours de l'exploration des couches d'incendies et de destructions évoquées plus haut dans la partie sud-ouest du secteur " $\mathrm{O}$ ", il a été découvert une série de complexes qui leur sont transversaux (des fosses domestiques et trois cabanes semi-enterrées) que le matériau en céramique trouvé dans leur comblement (pl. XXII) permet de dater du dernier quart et de la fin du VI e s. av. J.-C. Deux cabanes semi-enterrées de cette époque ont une forme en plan proche de l'ovale ( $\mathrm{n}^{\text {os }} 41$ et 42 ), une autre ( ${ }^{\circ} 49$, pl. XVII.2) est de plan rectangulaire. Dans cette même zone du territoire exploré a été découvert un autre ouvrage intéressant, qui remonte de toute évidence à la même époque et qu'on peut identifier comme une forge destinée au travail du fer. Cette construction se présente comme une fosse ( $\left.n^{\circ} 143\right)$, dont le plan de coupe est piriforme (fig. 8), d'une profondeur de près de $1,60 \mathrm{~m}$ et d'un diamètre maximal de $1,06 \mathrm{~m}$. Les parois de la fosse, évasées à leur base, étaient recouvertes d'une épaisse couche (jusqu'à 30-50 cm) d'un enduit argileux calciné sous l'effet de hautes températures. L'enduit a gardé l'empreinte de nombreux et volumineux morceaux de charbon de bois qui sont les restes de grosses bûches. Du côté oriental de la forge a été mis au jour dans l'enduit une conduite d'arrivée d'air d'une largeur d'environ 18-20 cm, longeant la paroi orientale depuis l'ouverture de la fosse jusqu'en bas, et débouchant dans la partie principale de la forge, tout au fond. Du côté opposé, occidental, on observe dans le fond de la fosse, près de la paroi, un renfoncement arrondi d'un diamètre de $28 \mathrm{~cm}$ et d'une profondeur de près de $19 \mathrm{~cm}$, dans lequel est fiché un lingot de fer arrondi reproduisant la même forme. Dans le comblement du volume principal de la forge, il a été trouvé une quantité significative de morceaux de charbon de bois, de scories de fer, de même que des morceaux oblongs de métal, qui sont de toute évidence des réserves de fer en barre. Une concentration particulièrement élevée de morceaux de fer a été mise au jour dans le fond de la fosse, où ils reposaient en une couche presque régulière, recouverts de couches de charbon et de morceaux d'enduit argileux d'une épaisseur allant jusqu'à $10 \mathrm{~cm}$. Cette trouvaille représente le premier complexe de production bien conservé découvert sur le territoire de l'établissement de Bérézan et destiné directement à l'obtention de fer épuré.

Dans l'Antiquité, le bas fourneau le plus élémentaire se présentait sous la forme d'une fosse, parfois surmontée de parois s'élevant au-dessus de sa surface, que l'on remplissait de couches de charbon de bois en 
Fig. 8 - Coupe d'un bas fourneau de la fin du VI e s. av. J.-C. et échantillons de barres de fer extraites de son comblement. 
combustion et de minerai. La température voulue dans le fourneau était atteinte grâce à un tirage naturel ou à des arrivées d'air spécialement conçues (comme c'était de toute évidence le cas dans l'ouvrage qui nous occupe, puisqu'un tel canal d'arrivée d'air a été mis au jour entre la surface et le fond de la fosse). Le cycle de production prenait de huit à douze heures, au cours desquelles les scories s'écoulaient dans le fond du four, où elles étaient, à leur tour, progressivement remplacées par le fer, plus lourd. L'éponge de fer que l'on retirait de ces fours contenait une quantité importante d'impuretés, c'est pourquoi elle était soumise à une nouvelle fonte, dans un four analogue, et au martelage. Ce cycle était répété une certaine quantité de fois, de manière à obtenir la densité et la pureté voulue et à éliminer les scories agrégées au métal. Le produit final de ce processus était un fer forgeable qui contenait encore une proportion non négligeable d'impuretés ${ }^{32}$. Etant donné que des barres de métal préalablement soumises au martelage ont été découvertes dans le comblement de l'ouvrage qui nous occupe, il est manifeste que ce fourneau était utilisé pour l'une des étapes intermédiaires permettant l'obtention du fer forgeable.

Il va sans dire que l'apparition, sur le territoire étudié, précisément dans le dernier quart du VI ${ }^{\mathrm{e}}$ s. av. J.-C., d'un ouvrage tel que ce bas fourneau, n'est pas fortuite; elle ajoute une touche intéressante au tableau de cette partie de l'établissement de Bérézan durant la période en question. Les ouvrages de production de ce genre, de très haute température, présentaient évidemment de très grands risques d'incendie; il est donc peu probable qu'ils aient été en service dans une zone d'habitation, aménagée de quartiers urbains. L'apparition d'un bas fourneau, simultanément à celle de cabanes semi-enterrées, n'a été possible que pendant la courte période qui a suivi la destruction de la majorité des constructions de ce quartier de la ville et qui a précédé la reconstitution du tissu urbain à la fin du $\mathrm{VI}^{\mathrm{e}}$ siècle et au début du $\mathrm{V}^{\mathrm{e}} \mathrm{s}$. av. J.-C. Le retour à la construction d'habitats semi-enterrés a été, selon toute vraisemblance, de très courte durée. Ces structures étaient des sortes de logements de fortune et ont précédé immédiatement la conduite dans cette même zone de grands travaux. Au tournant des $\mathrm{VI}^{\mathrm{e}}$ et $\mathrm{V}^{\mathrm{e}}$ siècles ou au début du $\mathrm{V}^{\mathrm{e}}$ s. av. J.-C., il a été construit sur ce territoire un complexe d'édifices peu ordinaires qui avaient, selon toute vraisemblance, une fonction publique.

32. J. F. Healy, Mining and Metallurgy in the Greek and Roman World, p. 184 sq. 
Le complexe d'édifices publics de la fin $d u V I^{e}$ siècle et du premier tiers $d u V^{e}$ s. av. J. $-C$.

Au cours des travaux des années 2008-2010, des vestiges de constructions de la fin du VI $\mathrm{V}^{\mathrm{e}}$ siècle et du premier tiers du Ve s. av. J.-C. ont été explorés dans la partie sud-ouest du secteur " $\mathrm{O}$ ", sur une surface d'environ $450 \mathrm{~m}^{2}$ (fig. 9). Un bâtiment (complexe $\mathrm{n}^{\circ}$ 1, pl. XXIII.1), composé de trois pièces $\left(\mathrm{n}^{\mathrm{os}} 9,10\right.$ et 11$)$, a été entièrement mis au jour; une autre construction (complexe $\mathrm{n}^{\circ} 2$, pl. XXIII.2), située à 5,4 m à l'ouest du complexe $\mathrm{n}^{\circ} 2$, a été partiellement explorée. Il a également été mis au jour une enceinte, qui délimite à l'est l'espace ouvert autour de ces constructions et les sépare de la rue de la ville. Les limites au sud, à l'ouest et au nord de ce territoire clos n'ont pas encore été dégagées à l'heure actuelle. Les particularités de construction des structures et des enceintes découvertes, de même que les données stratigraphiques, laissent penser que toutes ces bâtisses ont été érigées en une seule fois.

L'enceinte (mur 48) se présente sur presque toute sa longueur comme un socle de pierre d'épaisseur moyenne de $85-90 \mathrm{~cm}$, fait d'une couche de grandes dalles de calcaire grossièrement travaillées. A l'ouest de l'enceinte se trouve un bâtiment (complexe $\mathrm{n}^{\mathrm{o}} 1$ ) constitué de trois pièces. Bien que ses murs aient eux aussi été dans une large mesure démantelés dans l'Antiquité, les tranchées de construction permettent de reconstituer de manière assez sûre l'agencement du bâtiment.

Les fondations de la bâtisse étaient constituées de blocs de pierre arrondis d'origine volcanique, probablement arrivés à Bérézan comme ballast de navire. Ces blocs reposaient en deux rangées, par endroits en trois, et sur une seule couche de hauteur, dans une tranchée large de $60-70 \mathrm{~cm}$ et d'une profondeur allant jusqu'à $40 \mathrm{~cm}$; ils étaient recouverts d'une couche de sable pur. La partie du socle située au-dessus des fondations n'a été bien conservée que pour un seul mur du complexe, mais elle permet de reconstituer la construction des autres murs de l'édifice: les tranchées des fondations, où reposent les pierres de ballast et qui sont comblées de sable, étaient recouvertes de grosses dalles de calcaire grossièrement travaillées et posées à plat. Ces dalles servaient de base à un appareil d'orthostates à double parement disposés en trois épaisseurs constructives, fait de blocs de calcaire quasi rectangulaires, soigneusement taillés. L'espace entre les deux parements d'orthostates, qui formaient la façade du mur, était comblé d'un blocage en pierre finement 
Fig. 9 - Le secteur «O $\mathrm{O}$. Plan des vestiges de constructions de la fin du VI et du premier quart du $\mathrm{V}^{\mathrm{e}}$ s. av. J.-C.

concassée. La hauteur du socle du mur 42 atteint $38 \mathrm{~cm}$ à $50 \mathrm{~cm}$ pour une largeur d'environ $50-52 \mathrm{~cm}$.

Le complexe $\mathrm{n}^{\circ} 1$ (pl. XXIII.1) a un plan de forme proche du carré; ses dimensions sont de 11,45 m x 11,30 m. Sa partie nord est divisée en deux pièces de surface inégale (la pièce orientale $\mathrm{n}^{\mathrm{o}} 11$ a une surface de $16,32 \mathrm{~m}^{2}$, la pièce occidentale $\mathrm{n}^{\circ} 9$, de $26,68 \mathrm{~m}^{2}$ ); la cloison qui sépare ces deux pièces était un mur épais, qui reposait sur une assise de blocs de pierre placés dans une tranchée et qui ne comportait en tout cas pas d'embrasure de porte (les pièces orientale et occidentale ne communiquaient donc pas entre elles). La pièce sud du bâtiment ( $\left.\mathrm{n}^{\mathrm{o}} 10\right)$ n'avait pas de mur extérieur: elle était ouverte au sud. Aucune trace d'appareil ou de tranchée de construction qui relierait les extrémités sud des murs 46 et 54 n'a été mise au jour. Il est tout à fait possible qu'il s'agisse d'un portique ceint à l'ouest et à l'est de longues antae de longueur inégale 
(3,85 m pour l'oriental, 5,40 m pour l'occidental), bien que les fouilles n'aient permis de fixer aucune trace nette de bases de colonnes. Selon toute vraisemblance, l'ante occidentale, plus longue, était prolongée vers le sud par un mur d'un autre caractère (peut-être une enceinte), entièrement démoli, dont il ne reste qu'une tranchée peu profonde (sans traces de fondations en blocs de pierre) ainsi que des pierres isolées. Les vestiges de cette construction sont observables sur une longueur de 9,17 m, jusqu'à la limite sud du secteur étudié. La fondation de l'ante orientale (c'est-à-dire l'extrémité sud du mur 46) se présente sous la forme d'une construction de trois grandes dalles de calcaire.

Pour ce qui est du mur qui sépare la pièce sud des pièces orientale et occidentale, la partie de son socle qui émerge du sol a beaucoup souffert des récupérations de pierres et des fouilles clandestines; on ne peut donc que faire des suppositions quant à la présence d'ouvertures menant dans les deux pièces fermées. Aucun détail de l'intérieur des pièces du complexe n'a pu être mis au jour. Les sols étaient de toute évidence en terre; dans la pièce 9, on a pu identifier le niveau de damage du sol en argile. Dans la partie orientale de la pièce 10 ont été découverts deux amoncellements de morceaux de calcaire non travaillé de tailles diverses (sur une surface totale de près de $16 \mathrm{~m}^{2}$ ), qui constituent peut-être les vestiges d'un pavement.

La pièce sud porte les traces d'une reconstruction: on y a dégagé un mur ( $\mathrm{n}^{\circ} 57$ ), d'un type d'appareil irrégulier, qui sépare la pièce 10 en deux parties inégales. La tranchée de fondation caractéristique des autres murs du complexe n'a pas été mise au jour sous l'assise de cet appareil. Cette dernière se trouvant $8 \mathrm{~cm}$ à $19 \mathrm{~cm}$ au-dessus du niveau des sols du complexe, on peut affirmer qu'elle a été érigée au cours d'une transformation du complexe et qu'elle n'existait pas dans le plan initial. La fonction de cet appareil, qui s'interrompt vers le sud, reste incertaine.

A une distance de 5,40 m à l'ouest de la construction décrite, une bâtisse voisine (complexe $\mathrm{n}^{\circ} 2$, pl. XXIII.2) a été partiellement mise au jour. Ce deuxième complexe avait selon toute vraisemblance la même orientation dans l'espace et était construit selon un plan tout à fait semblable. Sa pièce occidentale n'a pu être mise au jour que partiellement durant les fouilles de 2010. La pièce orientale a une superficie de $32,90 \mathrm{~m}^{2}$, la longueur de l'édifice du nord au sud est de 9,8 m. Dans la pièce sud du deuxième complexe, à la différence du premier, les fondations en pierre de trois piliers de soutènement, placés à une distance de 
1,20 m l'un de l'autre, ont été conservées. Il est remarquable que dans le complexe de 2010, la pièce sud soit également divisée en deux parties inégales par un appareil plus tardif de destination inconnue, de manière tout à fait analogue au complexe contenant les pièces 9-11.

L'appareil d'orthostates des socles de ce bâtiment a été presque complètement démantelé dans l'Antiquité; des restes n'en ont été conservés que dans deux zones limitées. Le plan du bâtiment peut néanmoins être reconstitué grâce aux tranchées de fondation, grâce aussi à de grandes dalles horizontales de calcaire du premier rang de l'appareil des socles. Les murs du deuxième complexe étaient également sur fondations, mais celles-ci étaient de facture différente: des tranchées d'une profondeur de 50-60 cm étaient comblées de gravier calcaire damé qui assurait la fonction de "coussin" sous la base des socles en pierre des murs.

Comme l'ont montré les recherches de 2009, au sud du bâtiment comprenant les pièces $n^{\text {os }} 9-11$ s'étendait une vaste surface non construite, où des fragments d'un seul appareil (mur 59) ont été fixés. Selon les données stratigraphiques, ces vestiges de constructions devaient être chronologiquement très proches du bâtiment examiné plus haut, mais ils ne sont en aucune manière liés à ce dernier et appartiennent à un autre édifice. Dans l'espace compris entre les complexes $n^{\text {os }} 1$ et 2 a été découvert le lit d'un large réservoir et les restes d'un pavement, un damage très dense de gravier de calcaire.

Le plan inhabituel des édifices mis au jour, les particularités, uniques pour l'établissement de Bérézan, des techniques de construction adoptées pour leur édification, de même que le fait qu'ils soient situés à l'intérieur d'un terrain clos qui leur est spécialement destiné au sein d'un quartier de maisons d'habitation et que l'orientation des complexes $\mathrm{n}^{\text {os }} 1$ et 2 ne coïncident pas avec celle du réseau urbain environnant, tout cela amène à les considérer comme des bâtiments publics, un complexe d'édifices lié au culte ou à destination civile. Une interprétation plus précise de ces structures se heurte cependant pour l'instant au fait que leur mauvais état de conservation hypothèque la validité d'une reconstitution de leur architecture, ainsi qu'au manque de données quant à leur environnement. Sur le sujet, on ne peut à l'heure actuelle que se contenter de quelques suppositions.

Bien que les constructions à antes avec deux cellae soient rares dans l'architecture des temples grecs de la période archaïque tardive, on 
connaît tout de même quelques exemples d'édifices de ce genre. La plus étroite analogie avec nos complexes, au niveau du plan, peut être trouvée dans les deux constructions du sanctuaire (dédié vraisemblablement à Apollon) d'Aliki, sur la côte méridionale de Thasos, constructions explorées en $1963^{33}$. Elles se présentent comme des édifices d'ordre dorique de plan quasiment identique, mais de dimensions différentes. Leur orientation coïncide presque avec celle des complexes de Bérézan (avec une entrée au sud-ouest). Elles sont également très proches par leur datation: le bâtiment le plus ancien (celui du nord, le plus grand) a été érigé avec un portique ionique aux environs de 525 av. J.-C. (il a été reconstruit postérieurement en style dorique), et le bâtiment sud, au portique dorique, au tournant des $\mathrm{VI}^{\mathrm{e}}$ et $\mathrm{V}^{\mathrm{e}}$ s. av. J.-C. ${ }^{34}$. La façade du plus petit des bâtiments d'Aliki (le bâtiment sud) était ornée de cinq colonnes entre les antae; ses dimensions, $11,59 \mathrm{~m}$ d'est en ouest et $12,91 \mathrm{~m} \mathrm{du}$ nord au sud ${ }^{35}$, sont très proches de celles du complexe comprenant les pièces $n^{\text {os }}$ 9-11 de Bérézan.

Le principal espace intérieur des édifices d'Aliki était lui aussi divisé en deux pièces de superficie inégale; la plus grande des deux étant en outre, dans les deux bâtiments, la pièce ouest (nord-ouest). Au centre de ces pièces étaient placés des foyers de pierres rectangulaires de taille basse (selon la première publication, des autels eschara ${ }^{36}$ ).

De tels édifices sont identifiés à l'heure actuelle comme des «salles de banquet", des hestiatoria, constructions que l'on trouvait attenantes à de nombreux sanctuaires du monde grec et dans lesquelles se déroulaient les banquets sacrificiels qui accompagnaient les fêtes religieuses. En tant que bâtiment spécialisé séparé, l'hestiatorion est attesté par les données épigraphiques dès le tournant des $\mathrm{VI}^{\mathrm{e}}$ et $\mathrm{V}^{\mathrm{e}}$ s. av. J.-C. ${ }^{37}$. Ces édifices étaient selon toute vraisemblance destinés aux banquets d'un cercle restreint de participants aux cérémonies, composé des citoyens les plus éminents, de magistrats, de prêtres. Diverses variantes de plans sont connues pour ces bâtiments, du plus simple, comportant une ou deux pièces, à

33. G. Daux, "Chronique des fouilles 1963 », p. 885, fig. 1.

34. Ch. Börker, Festbankett und Griechische Architektur, p. 14 sq.

35. J. Servais, Aliki I: Les deux Sanctuaires, p. 49.

36. Ibid., p. 68.

37. Ch. Börker, Festbankett und Griechische Architektur, p. 11. 
une multitude de "salles de banquet" qui s'étendent en chaîne ou qui sont regroupées autour d'une cour commune ${ }^{38}$.

On connaît un hestiatorion très ressemblant par son plan à l'édifice de Bérézan, mais datant de la période hellénistique, dans le temple d'Héra de Perachora (à l'ouest du temple d'Héra Limenia); dans ce cas-là, la fonction des deux pièces communicantes a d'ailleurs été confirmée par la mise au jour de deux couches taillées dans la pierre et se trouvant en place ${ }^{39}$. Il est intéressant de noter que le bâtiment du sanctuaire à Héra était dépourvu de colonnes et que les deux pièces avec couches communiquaient par un vestibule auquel les visiteurs accédaient par une très large ouverture de porte dans la partie centrale de la façade. L'hestiatorion du sanctuaire d'Athéna Pronaia à Delphes, construit au $\mathrm{V}^{\mathrm{e}}$ s. av. J.-C., suit un plan de construction similaire ${ }^{40}$.

Dans l'architecture des villes antiques du nord de la mer Noire, il n'avait été auparavant dégagé quasiment aucun bâtiment public spécifiquement destiné aux festins. Il y a une exception, avec un édifice à une pièce du IVe s. av. J.-C., situé sur le site de Čajka et identifié par Sergej Anatol'evič Kovalenko comme un hestiatorion ${ }^{41}$. L'existence de constructions de ce type est toutefois très probable. Ainsi, plusieurs inscriptions sur des kylix du $\mathrm{V}^{\mathrm{e}}$ s. av. J.-C. provenant d'Olbia font mention d'un collège de Néomnèiastes, ce qui peut témoigner du fait que cette confrérie religieuse fêtait chaque mois la nouvelle lune, probablement en organisant un festin sacrificiel dans le temple d'Apollon Delphinios ${ }^{42}$.

Les dimensions de la pièce $9\left(5,80 \mathrm{~m}\right.$ x 4,60 $\left.\mathrm{m}=26,68 \mathrm{~m}^{2}\right)$ sont assez typiques des salles de banquet des sanctuaires antiques. Leur superficie et leur forme, proche du carré, étaient dictées par la nécessité d'installer le long du périmètre des murs des klinai, dont la longueur variait d'environ $1,50 \mathrm{~m}$ à un peu plus de $2 \mathrm{~m}^{43}$. Ainsi, la pièce occidentale du

38. Ibid., p. 24, Abb. 8.

39. R. A. Tomlinson, Greek Sanctuaries, p. 42-44, fig. 7 et "The Upper Terraces at Perachora".

40. Ch. Börker, Festbankett und Griechische Architektur, p. 25, Abb. 9.

4I. S. A. Kovalenko, "Gestiatorij čajkinskogo gorodišča».

42. A. S. Rusjaeva, Religija i kul'ty antičnoj Ol'vii, p. 50 ; M. V. Skržinskaja, Budni i prazdniki Ol'vii v VI-I vv. do n. è., p. 45 et 56.

43. S. A. Kovalenko, "Gestiatorij čajkinskogo gorodišča», p. 130. 
complexe nord d'Aliki, par exemple, a des dimensions d'environ $6,4 \mathrm{~m}$ x $6,3 \mathrm{~m}^{44}$; on peut y reconstituer l'installation de près de onze klinai.

Même si l'hypothèse de l'identification comme hestiatorion du bâtiment qui contient les pièces 9,10 et 11 est assez probable, on ne saurait la retenir de manière inconditionnelle. Dans la pièce 9, il n’a été fixé aucune trace d'installation de couches, de plate-forme ou de foyer central. Le décalage par rapport à l'axe central de l'ouverture de la porte serait un argument de poids en faveur de l'installation de klinai, mais, comme on l'a déjà signalé plus haut, il n'a pas été découvert d'ouverture de porte entre les pièces 9 et 11 et la pièce 10 , à cause du mauvais état de conservation du mur 55 qui les sépare. Cependant, d'après les vestiges d'appareil d'orthostates du socle du mur 55 sur un tronçon d'une longueur de 3,80 m depuis l'angle avec le mur ouest du complexe (pour une longueur totale du tronçon du mur 55 dans la pièce $\mathrm{n}^{\circ} 9$ égale à $5,80 \mathrm{~m}$ ), on peut supposer que si une ouverture de porte dans ce mur a jamais existé, elle devait se situer non pas au centre, mais décalée vers l'est.

Malheureusement, les trouvailles d'objets dans les pièces du complexe ne permettent pas non plus de préciser la fonction à laquelle il était destiné. Les ruines du bâtiment, selon toute vraisemblance, se sont élevées durant une assez longue période en surface, bien visibles, et ont été progressivement détruites, leurs pierres étant activement réemployées (à cause sans doute de l'excellente qualité des pierres taillées qui composaient l'appareil d'orthostates du socle). Il a été constaté au cours des fouilles que les niveaux de destruction, très chargés en coquilles de moules et en céramique du milieu et de la deuxième moitié du $\mathrm{V}^{\mathrm{e}} \mathrm{s}$. av. J.-C., ont "coulé» dans les pièces du complexe, atteignant par endroits le niveau du sol. Il n'a donc pas toujours été possible de distinguer de manière certaine les trouvailles appartenant à l'horizon des constructions du premier tiers du $\mathrm{V}^{\mathrm{e}}$ s. av. J.-C. des matériaux provenant des couches qui les ont recouvertes. On ne mentionnera particulièrement qu'un seul objet, qui peut être en rapport avec ce complexe bien qu'il n'ait pas été trouvé à l'intérieur, mais devant son entrée (à environ $10 \mathrm{~m}$ au sud de la pièce 10). Il s'agit d'un luminaire ouvert à trois sections, en marbre, présentant des orifices pour être suspendu (pl. XXIV.1, БЭ 2009 29/241). Cet objet peut être comparé à un autre objet du même type, découvert lors des fouilles de Milet et daté de la deuxième moitié

44. J. Servais, Aliki I: Les deux Sanctuaires, p. 50, fig. 57. 
ou de la fin du VII ${ }^{\text {s. }}$ s. av. J.-C. ${ }^{45}$. Le niveau d'enfouissement de l'objet correspond à l'horizon du niveau de circulation du complexe 1. La découverte d'un luminaire de ce type est extrêmement intéressante, car elle n'est pas caractéristique d'une construction d'habitation; de tels luminaires proviennent souvent de sanctuaires ${ }^{46}$. La différence de datation de la couche d'extraction et du luminaire, qui s'élève à plus de cent ans, permet de supposer qu'il a longtemps été conservé par les habitants de Borysthène, probablement durant plusieurs générations.

Le moment où les bâtiments décrits cessent d'être en fonction doit selon toute vraisemblance être situé au deuxième quart du Ve s. av. J.-C. Les recherches de longue haleine menées dans la partie nord de l'établissement de Bérézan par Ljudmila Vladimirovna Kopejkina, Jaroslav Vital'evič Domanskij et Sergej L'vovič Solov'jov ont conduit à supposer que le déclin de l'établissement se situe au $\mathrm{V}^{\mathrm{e}}$ s. av. J.-C., déclin qui s'est manifesté par la diminution de la surface habitée, la décroissance du nombre d'habitants, la dégradation des constructions urbaines et, corollaire, un changement spectaculaire de l'apparence de la ville ${ }^{47}$. Ce point de vue a été vigoureusement contesté par Lapin, qui s'est basé sur les résultats de ses travaux sur le secteur « $\mathrm{O}$ » dans la partie orientale de l'île pour formuler l'hypothèse d'un développement ininterrompu de l'établissement de Bérézan au cours des $\mathrm{V}^{\mathrm{e}}$ et $\mathrm{IV}^{\mathrm{e}} \mathrm{s}$. av. J.-C. D'après lui, c'est précisément au $\mathrm{V}^{\mathrm{e}} \mathrm{s}$. av. J.-C. que se rapportent la plus grande quantité de vestiges de construction et la plus grande expansion des frontières du territoire habité de la ville ${ }^{48}$. Les nouvelles recherches opérées dans le même secteur livrent cependant un tableau tout à fait différent.

Le territoire exploré a la particularité suivante: des couches de destruction $\mathrm{du} \mathrm{V}^{\mathrm{e}}$ s. av. J.-C. recouvrent les restes de construction en pierre et brique crue de la période archaïque tardive. Ces couches sont chargées d'une énorme quantité de coquillages de moules concassés, d'os d'animaux, de fragments de céramique fortement calcifiés. Le gros des trouvailles en matière de céramique, avant tout des restes d'amphores,

45. W. Selesnow, "Funde aus Milet XIII», p. 40, Abb. 11, L11.

46. J. D. Beazley, "A Marble Lamp».

47. L. V. Kopejkina, «Osobennosti razvitija poselenija na o. Berezan’ v arkhaičeskij period", p. 206-208; J. V. Domanskij, J. G. Vinogradov, S. L. Solov’jov, "Osnovnye rezul'taty rabot Berezanskoj èkspedicii " p. 38 ; S. L. Solovyov, Ancient Berezan, p. $98-113$.

48. V. V. Lapin, Grečeskaja kolonizacija Severnogo Pričernomor' ja, p. 108. 
permet de rapporter la couche aux trois premiers quarts du $\mathrm{V}^{\mathrm{e}}$ s. av. J.-C. On situe l'époque la plus probable de sa formation autour du second quart et de la moitié du $\mathrm{V}^{\mathrm{e}}$ s. av. J.-C. Ce qui frappe de prime abord à l'examen des découvertes faites dans les couches de destruction, c'est le très haut pourcentage de restes d'amphores (jusqu'à 90\%-96\% de la quantité totale de fragments de céramique) et la relative pauvreté des éléments d'autres groupes du complexe céramique. Les bâtiments abandonnés de la période précédente étaient alors en train de tomber progressivement en ruines, les pierres de leurs appareils étaient récupérées. Il n'a été mis au jour au cours des fouilles aucun reste de construction de grande envergure qui soit daté de la deuxième moitié du $\mathrm{V}^{\mathrm{e}}$ et du $\mathrm{IV}^{\mathrm{e}} \mathrm{s}$. av. J.-C., alors qu'il a été examiné quatre fosses d'implantation, pratiquées dans le sol pour des cabanes semi-enterrées, stratigraphiquement simultanées aux niveaux de destruction du $\mathrm{V}^{\mathrm{e}} \mathrm{s}$. av. J.-C. Le plus évocateur de ces complexes est une cabane semi-enterrée circulaire ( $\left.n^{\circ} 46\right)$, très ressemblante par sa forme et sa construction aux complexes analogues de la première moitié du VI ${ }^{\mathrm{e}}$ s. av. J.-C. Son diamètre est de 3,10-3,30 m pour une profondeur d'environ $80 \mathrm{~cm}$. Dans son fond se trouvaient sept trous de poteaux de diamètres divers: l'un pour le poteau central et six le long des parois (pl. XXIV.2). Le sol était enduit d’argile.

Etant donné que cette cabane semi-enterrée est apparue quasiment en face d'un bâtiment public de la fin du VI ${ }^{\mathrm{e}}-$ début du Ve s. av. J.-C. et qu'elle a été implantée dans une strate à partir du niveau des couches de destruction qui recouvraient les vestiges d'éléments de construction de cet édifice, il est clairement évident qu'elle a été creusée à une époque où le complexe $\mathrm{n}^{\mathrm{o}} 1$ n'existait plus. Des trouvailles évocatrices de céramique, faites dans le comblement du complexe bâti 46 (pl. XXV.1-4), nous donnent un convaincant terminus post quem pour la période d'activité des bâtiments publics plus anciens. La plus représentative d'entre elles est un groupe de céramique de table attique comprenant une série de fragments de kylix et de skyphos tardifs à figures noires, décorés dans la manière du peintre de Haimon (pl. XXV.1-2) et datés des années 490-480 av. J.-C. De nombreux fragments de kylix et de coupelles à vernis noir donnent une datation approchante. Ainsi, même si l'on tient compte du temps d'utilisation de la céramique peinte (certains objets découverts portent des traces de réparation), on peut situer l'époque d'existence de ces cabanes semi-enterrées autour des années 480-470 av. J.-C. 
Le retour à la tradition de construction d'habitations enterrées, qui semblait quasiment oubliée depuis le milieu du $\mathrm{VI}^{\mathrm{e}}$ s. av. J.-C., avait déjà été observé plus tôt dans la partie nord-ouest de l'île: tout un groupe de cabanes enterrées de constructions diverses a été dégagé au cours des fouilles des années 1987 à $1991^{49}$. On peut désormais affirmer que des processus semblables à ceux-là se déroulaient également au même moment dans la partie est de l'établissement. Malgré la dégradation des bâtiments urbains, les couches de destruction signalées témoignent d'une activité domestique intense dans le secteur étudié aux deuxième et troisième quarts du $\mathrm{V}^{\mathrm{e}} \mathrm{s}$. av. J.-C. La composition spécifique de céramique de ces couches, qui contiennent une quantité significative d'amphores du deuxième et du troisième quart du $\mathrm{V}^{\mathrm{e}}$ s. av. J.-C. (pl. XXV.6-7), pourrait confirmer indirectement le fait que Bérézan a servi de base d'ancrage de navires et de point de rupture de charge. Une découverte intéressante dans les couches de destruction est celle d'une grande pièce de monnaie en forme de dauphin (pl. XXV.5). Dans la mesure où ces découvertes de grandes pièces de monnaie sont très rares, le rapport entre leur valeur nominale et celle des habituelles petites monnaies en forme de dauphin n'a pas été établi avec certitude. Il est cependant à noter que le poids de notre exemplaire $(35 \mathrm{~g})$ est environ deux fois moindre que celui de deux grandes monnaies de dauphin connues, qui pèsent $71 \mathrm{~g}^{50}$.

Ainsi, les travaux de ces dernières années, menés par les archéologues du Musée d'Etat de l'Ermitage (Saint-Pétersbourg) dans la partie nordest de l'île de Bérézan, ont livré un volume conséquent de nouvelles données touchant à la chronologie du développement et à l'apparence de cet établissement grec archaïque. La datation du passage à la construction généralisée d'habitations non enterrées en pierre et brique crue est devenue plus précise (cette transition peut être située, nous semble-t-il, au début du troisième quart du VIe s. av. J.-C.). Des traces manifestes d'incendies et de destructions laissent supposer certaines catastrophes qui se seraient abattues sur l'établissement au dernier quart de ce même siècle. A cette lumière, les nombreuses traces de reconstruction du dernier quart et de la fin du VI e s. av. J.-C., observées auparavant dans la partie nordouest de l'île, prennent une nouvelle signification. Une découverte peut

49. S. L. Solovyov, Ancient Berezan, p. 101-109.

50. P. O. Karyškovskij, Monetnoe delo i denežnoe obraščenie Ol'vii (VI v. do n.è. - IV v. n.è.), p. 220, tabl. I = A 1-2. 
être considérée comme un résultat important des recherches menées, celle d'un complexe de bâtiments de plan et d'architecture inhabituels, dont le cadre de datation est la fin du $\mathrm{VI}^{\mathrm{e}}$ siècle et le premier tiers du $\mathrm{V}^{\mathrm{e}}$ s. av. J.-C. Le territoire qu'il occupe n'est pas encore complètement mis au jour à l'heure actuelle, ce qui, ajouté au fait que les restes de la construction sont en mauvais état de conservation, complique son interprétation. Le fait même de la présence d'un complexe d'édifices publics en fonction durant les dernières décennies de l'existence de la Borysthène archaïque tardive peut toutefois témoigner de la perpétuation des traditions de la polis parmi les habitants de l'établissement, qui faisait alors partie de l'Etat d'Olbia.

$$
\begin{array}{r}
\text { Dmitrij Efimovič ČIstov } \\
\text { Musée d'Etat de l'Ermitage, Saint-Pétersbourg }
\end{array}
$$

Traduction du russe de Mathilde ReIChler IMPERIALI 


\section{BIBLIOGRAPHIE}

Aydemir, Ahmet, "Funde aus Miletus XX. Kochgeschirr und Küchengeräte aus dem archaischen Milet", Archäologische Anzeiger, 2 (2005), p. 85-101.

Beazley, John D., "A Marble Lamp», Journal of Hellenic Studies, 60 (1940), p. 22-49.

Börker, Christoph, Festbankett und griechische Architektur, Konstanz, Universitätsverlag, 1983 (Xenia, Heft 4).

Bujsкikн, Alla Valer'evna, "Dejaki osoblyvosti planuval'noi struktury pizn'oarkhaičnykh poselen' Nyžn'ogo Pobužžja» ["Quelques particularités dans la structure des établissements archaïques tardifs du cours inférieur du Boug»], Arkheologija, 2 (1990), p. 24-32.

—, "O standartakh linejnykh mer v stroitel'no - kamenotesnom dele Khersonesa IV-II vv. do n.è.» [ A propos des standards de mesure linéaire dans la construction en pierre taillée de Chersonèse Taurique aux IV ${ }^{\mathrm{e}}-\mathrm{II} \mathrm{e}^{\mathrm{e}}$ siècles av. J.-C.»], Antičnyj Mir $i$ Arkheologija, 10 (1999), p. 95-100.

—, «Nekotorye polemičeskie zametki po povodu stanovlenija i razvitija Borisfena i Ol'vii v VI v. do n.è.» "Quelques remarques polémiques à propos de l'apparition et du développement de Borysthène et d'Olbia au VI ${ }^{\mathrm{e}}$ siècle av. J.-C.»], Vestnik Drevnej Istorii, 2 (2005), p. 146-164.

ČIstov, Dmitrij Efimovič, "Novyj kompleks s požariščem vtoroj poloviny VI v. do n.è. iz raskopok na učastke "O" antičnogo poselenija na o. Berezan” " [ Un nouveau complexe avec vestiges d'incendies de la seconde moitié du $\mathrm{VI}^{\mathrm{e}}$ siècle av. J.-C., issu des fouilles du secteur " $\mathrm{O}$ " de l'établissement antique sur l'île de Bérézan"], in Drevnosti Severnogo Pričernomor' ja $v$ antičnoe vremja [Antiquités du littoral septentrional de la mer Noire à l'époque classique], otv. red. Viktor Nikolaevič Zin'ko, Valentina Vladimirovna Krapivina, Simferopol', Krymskoe otdelenie Instituta vostokovedenija NANU i Centr archeologičeskih 
issledovanij BF «Demetra», 2007, p. 127-151 (Materialy po arkheologii, istorii i ètnografii Tavrii, Supplementum 4).

—, «K voprosu o poluzemljankakh arkhaičeskoj Berezani (po materialam raskopok na učastke “O” v 2004-2006 gg.) » [«Sur la question des cabanes semi-enterrées de la période archaïque de Bérézan (d'après les matériaux de fouilles du secteur " $O$ " en 2004-2006)»], in Drevnee Pričernomor'e [Le littoral pontique ancien], VIII, Odessa, 2008, p. 377-383.

—, "Novaja nakhodka fragmentov meča iz kompleksa serediny VI v. do n.è. na učastke "O” Berezanskogo poselenija» ["Nouvelle découverte de fragments d'une épée provenant d'un complexe du milieu du VIe siècle av. J.-C. situé dans le secteur " $O$ " de l'établissement de Bérézan"], in Bospor Kimmerijskij i varvarskij mir v period antičnosti i srednevekov'ja. Militaria [Le Bosphore Cimmérien et le monde barbare durant l'Antiquité et le Moyen Age. Militaria], otv. red. Viktor Nikolaevič Zin'ko, Kertch, 2008, p. 307-310 (Bosporskie čtenija IX).

Daux, Georges, "Chronique des fouilles 1963 ", Bulletin de correspondance hellénique, LXXXVIII (1964), p. 884-894.

Domanskij, Jaroslav Vital'evič, "Raboty Berezanskoj èkspedicii» ["Travaux de l'expédition de Bérézan»], in Arkheologičeskie Otkrytija 1983 g., Moskva, 1985, p. 272-273.

Domanskij, Jaroslav Vital'evič, Vinogradov, Jurij Germanovič, Solov'jov, Sergej L'vovič, "Osnovnye rezul'taty rabot Berezanskoj èkspedicii» " Principaux résultats des travaux de l'expédition de Bérézan"], in Itogi rabot arkheologičeskikh èkspedicij Gosudarstvennogo Ermitaža [Bilans des travaux des missions archéologiques du Musée d'Etat de l'Ermitage], otv. red. Galina Ivanovna Smirnova, Leningrad, Izdatel'stvo Gosudarstvennogo Ėrmitaža, 1989, p. 33-60.

Domanskij, Jaroslav Vital'evič, MarČEnKo, Konstantin Konstantinovič, "Bazovaja funkcija rannego Borisfena» [L'activité principale de la Borysthène primitive»], in Borysthenika-2004. Materialy meždunarodnoj naučnoj konferencii [Borysthenika-2004. Actes du colloque scientifique international], otv. red. Sergej Dmitrievič Kryžitskij, Nikolaev, Izdatel'stvo Nacional'nogo universiteta korablestroenija, 2004, p. 23-28. 
Healy, John F., Mining and Metallurgy in the Greek and Roman World, London, Thames and Hudson, 1978.

IL’INA, Julija Ivanovna, "O novykh nakhodkakh vostočno-grečeskoj keramiki na ostrove Berezan’" [ "A propos de nouvelles découvertes de céramique grecque orientale sur l'île de Bérézan»], in $\Sigma \Upsilon \Sigma \Sigma$ ITIA. Pamjati Jurija Viktoroviča Andreeva [ $\Sigma \Upsilon \Sigma \Sigma I T I A$. En mémoire de Jurij Viktorovič Andreev], otv. red. Vadim Jur'evič Zuev, Sankt-Peterburg, Aletejja, 2000, p. 201-209.

KaryšKovskij, Pjotr Osipovič, Monetnoe delo i denežnoe obraščenie Ol'vii (VI v. do n.è. - IV v. n.è.) [Monnayage et circulation monétaire à Olbia (VIe-IVe siècles av. J.-C.)], Odessa, Izdatel” "ČP Fridman A.S.», 2003.

Kopejkina, Ljudmila Vladimirovna, "Osobennosti razvitija poselenija na o. Berezan' v arkhaičeskij period (po rezul'tatam raskopok na severo-zapadnom učastke) » [ Particularités du développement de l'établissement de l'île de Bérézan durant la période archaïque (d'après les résultats des fouilles du secteur Nord-Ouest)»], Sovetskaja arkheologija, 1 (1981), p. 192-208.

—, «Rodossko-ionijskaja keramika VII v. do n.è. s ostrova Berezan' i ejo značenie dlja izučenija rannego ètapa suščestvovanija poselenija» [ "La céramique rhodo-ionienne du VII ${ }^{\mathrm{e}}$ av. J.-C. de l'île de Bérézan et sa signification pour l'étude de la période archaïque de l'établissement"], in Khudožestvennye izdelija antičnykh masterov [CEuvres d'art de maîtres de l'Antiquité], otv. red. Ksenija Sergeevna Gorbunova, Leningrad, Izdatel'tsvo «Iskusstvo», 1982, p. 6-36.

Kovalenko, Sergej Anatoljevič, "Gestiatorij čajkinskogo gorodišča " ["L'Hestiatorion du site de Čajka»], Vestnik drevnej istorii, 1 (2009), p. 119-135.

Krutilov, Valerij Viktorovič, "Železodelatel'naja masterskaja s syrcovymi stenami na territorii temenosa Berezanskogo poselenija» " Un atelier de sidérurgie aux murs de brique crue sur le territoire du temenos de la colonie de Bérézan"], in Drevnosti Severnogo Pričernomor'ja $v$ antičnoe vremja [Antiquités du littoral septentrional de la mer Noire à l'époque classique], otv. red. Viktor Nikolaevič Zin'ko, Valentina Vladimirovna Krapivina, Simferopol', Krymskoe otdelenie Instituta vostokovedenija NANU i Centr archeologičeskih issledovanij BF "Demetra", 
2007, p. 117-126 (Materialy po arkheologii, istorii i ètnografii Tavrii, Supplementum 4).

Lapın, Vladimir Vasil'evič, "Raskopki poselenija na ostrove Berezan' v 1960 g.» [ "Fouilles de l'établissement sur l'île de Bérézan en $1960 »]$, Kratkie soobščenija Instituta Arkheologii Akademii Nauk USSR, 11 (1961), p. 43-52.

—, Grečeskaja kolonizacija Severnogo Pričernomor'ja (Kritičeskie očerki otečestvennykh teorij kolonizacii) [La colonisation grecque du littoral septentrional de la mer Noire (aperçu critique des théories soviétiques de la colonisation)], Kiev, Naukova Dumka, 1966.

Mazarati, Svetlana Nikolaevna, OtrešKo, Valerij Mikhajlovič, "Poluzemljanki i zemljanki» ["Cabanes semi-enterrées et enterrées"], in Kul'tura naselenija Ol'vii i ee okrugi v arkhaičeskoe vremja [Le faciès culturel de la population d'Olbia et de ses environs à l'époque archaïque], otv. red. Sergej Dmitrievič Kryžitskij, Kiev, Naukova Dumka, 1987, p. 8-27.

Nazarov, Vladimir Vladmirovič, Gidroarkheologičeskaja karta černomorskoj akvatorii Ukrainy. Pamjatniki antičnoj i srednevekovoj èpokh [Carte hydro-archéologique des plans d'eau de la région de la mer Noire ukrainienne. Monuments de l'Antiquité et du Moyen Age], Kiev, Stilos, 2003.

Nazarov, Vladimir Vladmirovič, Kuz'MiščEv, Aleksandr Gennad'evič, "K probleme interpretacii odnoj pozdnearkhaičeskoj poluzemljanki na o. Berezan" " "Problème d'interprétation d'une cabane semi-enterrée de la période archaïque tardive découverte sur l'île de Bérézan»], in Bosporskij fenomen. Problema sootnošenija pis'mennykh i arkheologičeskikh istočnikov. Materialy meždunarodnoj naučnoj konferencii [Le phénomène bosporan. Problème de correspondance des sources écrites et archéologiques. Actes $d u$ colloque scientifique international], otv. red. Marina Jur'evna Vakhtina, Vadim Jur'evič Zuev, Sergej Vladimirovič Kašaev, Ol'ga Jur'evna Sokolova, Vladimir Andreevič Khršanovskij, Sankt-Peterburg, Izdatel'stvo Gosudarstvennogo Ėrmitaža, 2005, p. $178-180$.

Rusjaeva, Anna Stanislavovna, «Milet - Didimy - Borisfen - Ol'vija : Problemy kolonizacii Nižnego Pobuž ja” ["Milet - Didymes Borysthène - Olbia: Problèmes de la colonisation du cours inférieur du Boug»], Vestnik Drevnej Istorii, 2 (1986), p. 25-64. 
—, Religija i kul'ty antičnoj Ol'vii [Religion et cultes d'Olbia dans l'Antiquité], Kiev, Naukova Dumka, 1992.

Selesnow, Wolfgang, "Funde aus Milet XIII. Marmor- und andere Steinlampen", Archäologischer Anzeiger, 1 (2002), p. 27-40.

Servais, Jean, Aliki I: Les deux sanctuaires, Paris, Ecole française d'Athènes, 1980 (Etudes Thasiennes IX).

SkržInskaja, Marina Vladimirovna, Budni i prazdniki Ol'vii v VI-I vv. do n.è. [Vie quotidienne et fêtes à Olbia aux VI $I_{-} I^{e r}$ siècles av. J.-C.], Sankt-Peterburg, Aletejja, 2001.

Solov’Jov, Sergej L'vovič, «O formirovanii gorodskoj i polisnoj struktury Berezanskogo poselenija» [ «A propos de la formation de la structure urbaine et de la structure de polis de l'établissement de Bérézan»], Peterburgskij Arkheologičeskij Vestnik, 3 (1993), p. 39-43.

—, «Novye aspekty istorii i arkheologii antičnoj Berezani» ["Nouveaux aspects de l'histoire et de l'archéologie de la Bérézan antique»], Peterburgskij arkheologičeskij Vestnik, 8 (1994), p. 85-95.

—, «Iz istorii polisov Nižnego Pobuž ja: Borisfen i Ol'vija» ["De l'histoire des poleis du cours inférieur du Boug: Borysthène et Olbia»], in Antičnoe Pričernomor'e. Sbornik statej po klassičeskoj arkheologii, [Le littoral antique de la mer Noire. Recueil d'articles sur l'archéologie classique], otv. red. Sergei L'vovič Solov' jov, Sankt-Peterburg, Izdatel'tsvo Gosudarstvennogo Ėrmitaža, 2000, p. 94-111.

—, «Načalo antičnoj èpokhi v Severnom Pričernomor'e» [ «e début de l'époque antique dans la région septentrionale de la mer Noire»], in Borisfen - Berezan'. Načalo antičnoj èpokhi v Severnom Pričernomor'e. K 120-letiju arkheologičeskikh raskopok na ostrove Berezan' [Borysthène - Bérézan. Le début de l'époque antique sur le littoral de la mer Noire. A l'occasion du $120^{e}$ anniversaire des fouilles archéologiques sur l'île de Bérézan], otv. red. Mikhail Borisovič Piotrovskij et al., Sankt-Peterburg, Izdatel'stvo Gosudarstvennogo Ėrmitaža, 2005, p. 6-24.

Solovyov, S[ergei] L['vovič ], Ancient Berezan. The Architecture, History and Culture of the first Greek Colony in the Northern Black Sea, Leiden, Brill, 1999 (Colloquia Pontica 4).

Sparkes, Brian A., Talcott, Lucy, Black and plain pottery of the 6th, 5th, and 4th centuries B.C., Princeton, New Jersey, The American School of Classical Studies at Athens, 1970 (Athenian Agora 12). 
Tomlinson, Richard A., Greek Sanctuaries, London, Paul Elek, 1976.

-, "The Upper Terraces at Perachora", Annual of the British School at Athens, 72 (1977), p. 197-202.

Vinogradov, Jurij Germanovič, Političeskaja istorija Ol'vijskogo polisa [Histoire politique de la polis d'Olbia], Moskva, Nauka, 1989.

Vinogradov, Jurij Germanovič, Domanskij, Jaroslav Vital'evič, MarČenko, Konstanin Konstantinovič, "Sources écrites et archéologiques du Pont Nord-Ouest. Analyse comparative", in Le Pont-Euxin vu par les Grecs. Sources écrites et archéologie, textes choisis et présentés par Otar Lordkipanidzé et Pierre Lévêque, Paris, Les Belles Lettres, 1990, p. 121-139 (Annales Littéraires de l'Université de Besançon 427).

\section{Crédits iconographiques}

Toutes les photographies des vues des fouilles et des découvertes utilisées pour illustrer cet article ont été prises par Dmitrij Efimovič Čistov. Les objets découverts sont conservés au Parc national d'histoire et d'archéologie «Olbia» de l'Académie nationale des sciences d'Ukraine et au Musée militaire et historique A. V. Suvorov d'Očakovo. 